Pesq. Vet. Bras. 38(2):277-284, fevereiro 2018

\title{
Surtos consecutivos ocasionados por Eimeria zuernii em bezerros de corte de uma propriedade do estado de São Paulo ${ }^{1}$
}

\author{
Leonardo B. Cruvinel2 ${ }^{2}$, Thiago S.A. Bastos², João E. Nicaretta² ${ }^{2}$ Luiz F.M. Couto², \\ Dyego G.L. Borges ${ }^{3}$, Fernando A. Borges ${ }^{3}$, Vando E. Soares ${ }^{4}$ e Welber D.Z. Lopes ${ }^{2,5 *}$
}

\begin{abstract}
Cruvinel L.B., Bastos T.S.A., Nicaretta J.E., Couto L.F.M., Borges D.G.L., Borges F.A., Soares V.E. \& Lopes W.D.Z. 2018. [Consecutive outbreaks caused by Eimeria zuernii in calves on a rural property located in the municipality of São João da Boa Vista, State of São Paulo, Brazil.] Surtos consecutivos ocasionados por Eimeria zuernii em bezerros de corte de uma propriedade no estado de São Paulo. Pesquisa Veterinária Brasileira 38(2):277-284. Departamento de Medicina Veterinária Preventiva, Escola de Veterinária e Zootecnia, Universidade Federal de Goiás, Campus Samambaia, Avenida Esperança s/n, Campus Universitário, Goiânia, GO 74690.900, Brazil. E-mail: wdzlopes@hotmail.com

We report consecutive outbreaks (August 2015 and 2016) of disease caused by Eimeria zuernii in Canchim and Nelore calves about 45 days of age on a rural property in the municipality of São João da Boa Vista, state of São Paulo, Brazil. The first outbreak infected 78 calves ( 32 Nelore and 46 Canchim) between 35 and 55 days of age. The second outbreak infected 66 calves (36 Nelore and 47 Canchim) between 30 and 45 days of age. Some calves had bloody diarrhea and were dehydrated. Feces were collected from all animals (cows and calves) for coproparasitological examination and quantification of parasite eggs and oocysts per gram. The consecutive outbreaks in the 30 to 55 day-old calves occurred during the dry season (August). These outbreaks were caused by a high degree of infection by E. zuernii. Clinical signs (bloody diarrhea) and mortality of the calves triggered by eimeriosis/dehydration occurred in 8.3 and $1.4 \%$ of the herd in 2015 and 2016, respectively. These results highlight the importance of conducting field studies to better understand the parasitic dynamics of E. zuernii in calves younger than three months. Identifying the sources of infection, such as the water ingested by the animals, is also necessary, so that practical husbanddry can be recommended and adopted by the producers, to reduce the damage caused by outbreaks of Eimeria spp. in cattle herds.

INDEX TERMS: Calves, eimeriosis, Eimeria zuernii, parasitoses.
\end{abstract}

RESUMO.- 0 presente trabalho tem como objetivo relatar surtos consecutivos (Agosto de 2015 e 2016) ocasionado por Eimeria zuernii em bezerros de corte, Canchim e Nelore,

\footnotetext{
${ }^{1}$ Recebido em 13 de março de 2017.

Aceito para publicação em 9 de maio de 2017.

${ }^{2}$ Centro de Parasitologia Veterinária, Escola de Veterinária e Zootecnia, Universidade Federal de Goiás (UFG) - Campus Samambaia, Avenida Esperança s/n, Campus Universitário, Goiânia, GO 74.690.900, Brasil.

${ }^{3}$ Departamento de Medicina Veterinária, Universidade Federal do Mato Grosso do Sul (UFMS), Av. Filinto Müller 2443, Ipiranga, Cx. Postal 549, Campo Grande, MS 79074-460, Brasil.

${ }^{4}$ Universidade Brasil, Campus de Descalvado, São Paulo, SP 13690-970, Brasil.

${ }^{5}$ Departamento de Parasitologia, Instituto de Patologia Tropical e Saúde Pública (IPTSP), Universidade Federal de Goiás (UFG), Rua 235 s/n, Setor Leste Universitário, Goiânia, GO 74605-050. *Autor para correspondência: wdzlopes@hotmail.com
}

com aproximadamente 45 dias de vida, em uma propriedade rural localizada no município de São João da Boa Vista, estado de São Paulo. 0 primeiro surto foi diagnosticado em um lote composto por 78 bezerros, entre 35 e 55 dias de idade. Dos 78 bezerros, 32 eram Nelore e os outros 46 Canchim. Já o segundo, ocorreu em um novo lote de 66 bezerros (sendo 36 Nelore e 47 Canchim), com idade entre 30 e 45 dias. Alguns animais apresentaram diarreia sanguinolenta e desidratação. Colheitas de fezes de todos os animais (vacas e bezerros) foram realizadas para realização de exame coproparasitológico e quantificação de ovos e oocistos por grama (OPG e OoPG) de fezes de parasitos. Com base nos resultados encontrados neste estudo, é possível afirmar que os surtos consecutivos em bezerros de corte entre 30 e 55 dias de idade, na época seca do ano (agosto), foram desencadeados pelo elevado grau de infecção destes animais por E. zuernii. 
Analisando os dois anos em que os surtos ocorreram, a presença de sinais clínicos (diarreia sanguinolenta) e a mortalidade de bezerros desencadeada pela eimeriose/desidratação, aconteceram em 8,3\% 1,4\% do rebanho, respectivamente. Estes resultados destacam a importância em se realizar pesquisas de campo, com o objetivo de se melhor entender a dinâmica de parasitismo de E. zuernii e E. bovis em bezerros com idade inferior a três meses, em função dos motivos já discutidos neste estudo. Além disso, é necessário que se investigue as possíveis fontes de infecção, como a água ingerida pelos animais, para que condutas práticas possam ser adotadas/recomendadas aos proprietários, diminuindo desta maneira os prejuízos ocasionados por surtos de Eimeria spp em rebanhos bovinos.

TERMOS DE INDEXAÇÃO: Bezerros, eimeriose, Eimeria zuernii, parasitoses.

\section{INTRODUÇÃO}

Os bovinos em seu primeiro ano de vida estão susceptíveis a vários tipos de infecções que podem causar doenças ou até mesmo levar os animais a óbito. Problemas respiratórios e intestinais são os mais comuns, sendo que estes últimos, geralmente desencadeiam diarreia que por sua vez leva à perda de peso e atraso no crescimento, fatores estes que somados podem resultar em importantes perdas econômicas (Lopes et al. 2014).

Dentre as enfermidades intestinais, destaca-se a eimeriose cujos agentes são protozoários pertencentes ao filo Apicomplexa, classe Coccidia e gênero Eimeria spp. e compreende 12 espécies relacionadas à infecção mista de bovinos (Koutny et al. 2012). Esta enfermidade apresenta distribuição mundial, sendo responsável por significativas perdas econômicas no rebanho. Matjila \& Penzhorn (2002) estimaram prejuízos de aproximadamente US\$ 400 milhões de dólares/ano em bovinos nos USA, sem levar em consideração os casos subclínicos da enfermidade.

Apesar de existirem 12 espécies deste coccídio descritas, Eimeria bovis e E. zuernii são consideradas as mais patogênicas, responsáveis pelos quadros clínicos de eimeriose em bovinos (Bruhn et al. 2011). De acordo com Lima (2004), Taubert et al. (2008), Bruhn et al. (2011) e Koutny et al. (2012), estas duas espécies citadas anteriormente, dependendo da categoria e do grau de infecção, podem causar severos danos aos animais, ou até mesmo mortalidade de bovinos nas categorias mais susceptíveis. Estes autores destacam, ainda, que os casos clínicos mais severos são mais comuns de acontecerem nos animais entre três e seis meses, com aptidão leiteira, em função dos sistemas criação destes animais serem mais intensivos, em comparação aos de bovinos de corte.

Este trabalho tem como objetivo relatar a presença de surtos consecutivos ocasionados por Eimeria zuernii em bezerros de corte, com aproximadamente 45 dias de vida, em uma propriedade rural localizada no município de São João da Boa Vista, estado de São Paulo.

\section{MATERIAL E MÉTODOS}

Os surtos aconteceram na fazenda Santa Helena, uma propriedade rural localizada no município de São João da Boa Vista, estado de São Paulo, situada aproximadamente $220 \mathrm{~km}$ da capital, São Paulo. 0 município apresenta um relevo montanhoso, e o clima é tropical quente. A pluviosidade está em aproximadamente $1.140 \mathrm{~mm}^{3}$ anuais, e as chuvas concentram-se nos meses mais quentes, a partir de outubro até março. A temperatura média é de $28^{\circ} \mathrm{C}$, máxima $34^{\circ} \mathrm{C} \mathrm{e}$ mínima de $5^{\circ} \mathrm{C}$. No verão a média é de $22^{\circ} \mathrm{C}$ e no inverno de $18^{\circ} \mathrm{C}$ (disponível em www.climatempo.com.br).

$\mathrm{Na}$ fazenda onde foram diagnosticados os surtos consecutivos, existem aproximadamente 1.600 bovinos (Canchim e Nelore), que são criados em sistemas de cria, recria e engorda. Os animais pertencentes às fases de cria e recria ficam dispostos em pastos compostos por Brachiaria decumbens, enquanto que os animais de engorda realizam tal processo em confinamentos presentes na própria fazenda. A referida propriedade apresenta um alto grau de tecnificação, com um período de estação de monta, e a reprodução acontece por meio de inseminação artificial em tempo fixa (IATF), sendo que algumas matrizes recebem até três protocolos de IATF durante a referida estação.

Primeiro surto (agosto de 2015). 0 primeiro surto foi diagnosticado em 21 de agosto de 2015 em um lote composto por 78 bezerros, entre 35 e 55 dias de idade, que ficavam com suas respectivas mães. Dos 78 bezerros, 32 eram Nelore e os outros 46 Canchim. Já as matrizes eram compostas basicamente por vacas Canchim. É importante frisar que os surtos aconteceram apenas nos bezerros. Estes animais viviam com suas respectivas mães em uma taxa de lotação de aproximadamente 0,7 unidade animal (UA/hectare) por hectare.

Durante este primeiro surto, oito bezerros (sendo 5 Nelore e 3 Canchim) apresentaram diarreia sanguinolenta, com a presença de sangue vivo, sendo que dois destes animais estavam desidratados "olho fundo" (Figura 1AB). Durante a anamnese verificou-se que este episódio já era recorrente na propriedade a pelo menos três anos, sempre na mesma época do ano, e o tratamento para os casos de diarreia era realizado com doxiciclina (Corta Curso ${ }^{\circledR}$, Ouro Fino Agronegócios) ou sulfa + trimetropim (Borgal ${ }^{\circledR}$, MSD Saúde Animal). Os funcionários da fazenda diziam que o quadro clínico dos animais enfermos que recebiam estas medicações, apresentava uma melhora pouco significativa e, que em algumas situações, alguns bezerros vinham a óbito. Inspecionando o pasto, pode-se verificar que a apesar de seca, as pastagens encontravam-se com uma altura relativamente elevada ("alta") para a época do ano, e os bebedouros eram compostos por nascentes e lagoas represadas de cursos de água das nascentes que percorriam a fazenda.

Neste mesmo dia foi realizada a colheita de fezes de todos os animais (vacas e bezerros) para realização de exame coproparasitológico e quantificação de ovos e oocistos por grama (OPG e OoPG) de fezes de parasitos, de acordo com a técnica descrita por Gordon \& Whitlock (1939) modificado Ueno \& Gonçalves (1998).

Após a constatação de oocistos de Eimeria spp e ovos de helmintos (estrongilídeos) nos animais, as amostras de fezes foram subdivididas por categorias (bezerros e vacas), e as que apresentaram 0 oPG e $0 P G \geq 50$ foram reunidas em dois pools, sendo um para identificação das espécies de Eimeria spp., e outro para identificação genérica dos helmintos gastrintestinais (estrongilídeos) presentes nos bovinos.

Em relação à identificação específica dos oocistos de Eimeria spp, um destes pools (bezerros e vacas) foi processado 
utilizando-se o método de centrifugo-flutuação em solução de açúcar. Em seguida, o conteúdo foi filtrado utilizando-se peneira com gaze dobrada e o filtrado posteriormente acrescido de solução de dicromato de potássio $\left(\mathrm{K}_{2} \mathrm{Cr}_{2} \mathrm{O}_{7}\right) 2 \%$, volume a volume e mantidas a $24^{\circ} \mathrm{C}$ durante 14 dias sob oxigenação (bomba oxigenadora para aquários), para estimular a esporulação dos oocistos em laboratório. Os oocistos foram recuperados por centrifugação em solução saturada de açúcar à $60 \%$ e aproximadamente 100 oocistos por pool (de cada categoria animal, bezerros e vacas) foram identificados, utilizando-se microscópio de luz com sistema computadorizado LAS Leica ${ }^{\circledR}$. Quando possível, este processo de recuperação de 100 oocistos por pool para identificação das espécies de Eimeria spp. foi realizado em triplicata. A diferenciação entre as espécies foi realizada de acordo com as características fenotípicas dos oocistos, como cor, presença ou ausência de micrópila, comprimento e largura (Daugschies \& Najdrowski 2005).

No pool restante (de bezerros e vacas), realizou-se a coprocultura (Roberts \& O’Sullivan 1950). Para a identificação destas larvas $\left(\mathrm{L}_{3}\right)$ foi utilizado os critérios taxonômicos descritos por Keith (1953) e Costa (1982).

No mesmo dia em que o surto foi diagnosticado, todos os bezerros foram pesados e receberam toltrazuril $15 \mathrm{mg} / \mathrm{kg}$ via oral (Isocox ${ }^{\circledR}$, Ouro Fino Agronegócios), via oral, com o objetivo de eliminar Eimeria spp destes animais. Quatorze e 28 dias pós-tratamento (DPT), colheitas de fezes foram realizadas individualmente de cada animal (bezerros e vacas), para quantificação de oocistos e ovos por grama de fezes de parasitos, de acordo com a técnica descrita por Gordon \& Whitlock (1939) modificado Ueno \& Gonçalves (1998). As técnicas para identificação das espécies de Eimeria spp e genérica dos helmintos presentes nos animais, também foram aplicadas no $14^{\circ}$ e $28^{\circ}$ DPT, conforme metodologia descrita anteriormente.

Durante os 28 dias pós-tratamento, todos os bezerros foram observados quanto à presença de diarreia, com ou sem a presença de sangue e desidratação, com o objetivo de verificar quando os sinais clínicos cessariam após o tratamento específico com toltrazuril.

Antes das análises, as contagens de OPG e OoPG foram transformadas em logaritmo neperiano [ln(contagens de OoPG ou OPG+1)]. Após transformação, os dados foram submetidos aos testes de normalidade, homogeneidade e aleatoriedade dos erros. Posteriormente foram analisados em um esquema fatorial em parcela subdividida no tempo, onde, parcela foram os tratamentos (categoria animal). As confrontações das médias entre as diferentes datas de colheita foram aferidas pelo teste $t$ ao nível de 95\% de confiança (Sas 2002).

Segundo surto (agosto de 2016). Em 14 de agosto de 2016, o proprietário da fazenda, em contato telefônico, relatou haver os mesmos sintomas que os observados no surto anterior em um novo lote de 66 bezerros (sendo 36 Nelore e 47 Canchim), com idade entre 30 e 45 dias. Ao chegar à fazenda, pode-se constatar que estes animais estavam na mesma área (pasto) em que os bezerros de 2015 ficaram. Neste caso, a presença de diarreia sanguinolenta (sangue vivo) pode ser visualizada em quatro bezerros, sendo três Canchim e um Nelore. Dois destes animais também estavam desidratados.

Imediatamente foi realizada a colheita de fezes de todos os animais deste lote (vacas e bezerros) para realização de exame coproparasitológico (OPG e OoPG).

Concomitante a colheita de fezes, todos os bezerros foram pesados e tratados com toltrazuril (15mg/kg), pela via oral. No caso deste segundo surto houve apenas a colheita de fezes realizada no dia da visita, entretanto, nos 14 dias posteriores ao tratamento, todos os bezerros foram observados quanto à presença de diarreia, com ou sem a presença de sangue, e desidratação com o objetivo de verificar quando os sinais clínicos cessariam após o tratamento com toltrazuril.

\section{RESULTADOS}

Primeiro surto. Em relação aos resultados das contagens de OPG encontradas nos bezerros, no primeiro dia de visita do primeiro surto (dia zero), os exames revelaram que dos 78 animais, apenas cinco (6,4\%; contagem média de 11,99 OPG) encontravam-se infectados por helmintos estrongilídeos. Nas duas datas subseqüentes (14 e 28 dias pos-tratamento dos animais com toltrazuril), o grau de infecção médio dos bezerros por helmintos foi de 39,64 e 141,27 OPG, respectivamente (Quadro 1). Os resultados da coprocultura revelaram a presença de quatro gêneros, com os seguintes percentuais de infecção (ordem decrescente): Haemonchus (52,2\%), Cooperia (32,6\%), Oesophagostomum $(12,5 \%)$ e Trichostrongylus $(2,7 \%)$.

Para as vacas, as contagens médias de OPG obtidas no dia 0 (primeira visita no primeiro surto), 14 e 28, foram de 39,74, 8,97 e 4,48, respectivamente (Quadro 1). Pela coprocultura, foram identificadas larvas de Haemonchus $(67,8 \%)$, Cooperia $(14,8 \%)$ e Oesophagostomum $(17,4 \%)$.

Quadro 1. Análise estatística referente às contagens de ovos por grama de fezes (OPG, estrongilídeos) de fezes presentes nos bezerros e vacas nos primeiro e no segundo surto

\begin{tabular}{|c|c|c|c|c|c|c|c|}
\hline \multirow{3}{*}{ Lote } & \multicolumn{6}{|c|}{ Dia do estudo /Contagens médias ${ }^{\mathrm{a}}$ de ovos por grama (OPG, estrongilídeos) de fezes } & \multirow{3}{*}{$\operatorname{Pr}>F^{b}$} \\
\hline & \multicolumn{2}{|c|}{ Dia 0 (dia do início do surto) } & \multicolumn{2}{|l|}{14} & \multicolumn{2}{|l|}{28} & \\
\hline & Contagens médias & $\begin{array}{l}\text { Amplitude } \\
\text { de infecção }\end{array}$ & Contagens médias & $\begin{array}{l}\text { Amplitude } \\
\text { de infecção }\end{array}$ & Contagens médias & $\begin{array}{l}\text { Amplitude } \\
\text { de infecção }\end{array}$ & \\
\hline \multicolumn{8}{|c|}{ 1ㅇ Surto diagnosticado em agosto de 2015} \\
\hline Bezerros & 6,4 B & $(0-150)$ & $23,1 \quad \mathrm{~B}$ & $(0-350)$ & $109,6 \mathrm{~A}$ & $(0-950)$ & $<0,00001$ \\
\hline Vacas & $39,7 \mathrm{~A}$ & $(0-300)$ & $9,0 \quad \mathrm{~A}$ & $(0-100)$ & $4,48 \mathrm{~A}$ & $(0-100)$ & $<0,85431$ \\
\hline
\end{tabular}

2o Surto diagnosticado em agosto de 2015

$\begin{array}{lrr}\text { Bezerros } & 5,3 & (0-150) \\ \text { Vacas } & 42,4 & (0-300)\end{array}$

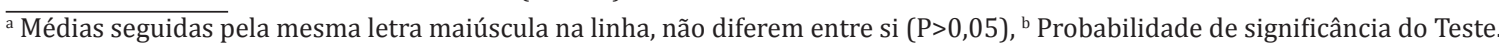


É importante lembrar que as vacas não receberam nenhum tipo de medicamento contra Eimeria spp. ou helmintos durante estas três datas de avaliação citadas anteriormente.

No que diz respeito às contagens de 0oPG para Eimeria spp., diagnosticada nos bezerros, foi possível observar que 70,51\% (55) dos animais estavam infectados pelo coccídio em questão. A contagem de OoPG mais elevada (61.550) foi diagnosticada em um bezerro que aprsentou diarréia sanguinolenta, e fragmentos de mucosa intestinal eliminadas junto as fezes (Quadro 2, Fig.1 e 2). A contagem média de OoPG para Eimeria spp diagnosticada para estes 78 bezerros nesta primeira etapa de exames, foi de 4.417,9. Após o tratamento dos animais com toltrazuril, as contagens de OoPG dos bezerros diminuíram significativamente $(P \leq 0,05)$, em comparação ao dia que o

Quadro 2. Análise estatística referente às contagens de oocistos por grama (OoPG, Eimeria) de fezes presentes nos bezerros e vacas nos primeiro e no segundo surto

\begin{tabular}{|c|c|c|c|c|c|c|c|}
\hline \multirow{3}{*}{ Lote } & \multicolumn{6}{|c|}{ Dia do estudo /Contagens médias ${ }^{a}$ de oocistos por grama (OoPG, Eimeria) de fezes } & \multirow{3}{*}{$\operatorname{Pr}>F^{b}$} \\
\hline & \multicolumn{2}{|c|}{ Dia 0 (dia do início do surto) } & \multicolumn{2}{|c|}{14} & \multicolumn{2}{|c|}{28} & \\
\hline & $\begin{array}{l}\text { Contagens } \\
\text { médias }\end{array}$ & Amplitude de infecção & $\begin{array}{l}\text { Contagens } \\
\text { médias }\end{array}$ & $\begin{array}{l}\text { Amplitude de } \\
\text { infecção }\end{array}$ & $\begin{array}{l}\text { Contagens } \\
\text { médias }\end{array}$ & $\begin{array}{l}\text { Amplitude de } \\
\text { infecção }\end{array}$ & \\
\hline \multicolumn{8}{|c|}{ 1은 Surto diagnosticado em agosto de 2015} \\
\hline Bezerros & 4417,9 A & $(0-61.550)$ & $14,7 \mathrm{~B}$ & $(0-250)$ & 123,4 B & $(0-4050)$ & $<0,00001$ \\
\hline Vacas & $1,9 \mathrm{~A}$ & $(0-50)$ & $1,3 \mathrm{~A}$ & $(0-50)$ & $1,92 \mathrm{~A}$ & $(0-50)$ & $<0,00001$ \\
\hline \multicolumn{8}{|c|}{$2^{\circ}$ Surto diagnosticado em agosto de 2015} \\
\hline Bezerros & 4308,9 & $(0-78.950)$ & & & - & & \\
\hline Vacas & 6,1 & $(0-150)$ & & & - & & \\
\hline
\end{tabular}

$\overline{{ }^{a}}$ Médias seguidas pela mesma letra maiúscula na linha, não diferem entre si $(\mathrm{P}>0,05),{ }^{\mathrm{b}}$ Probabilidade de significância do Teste.
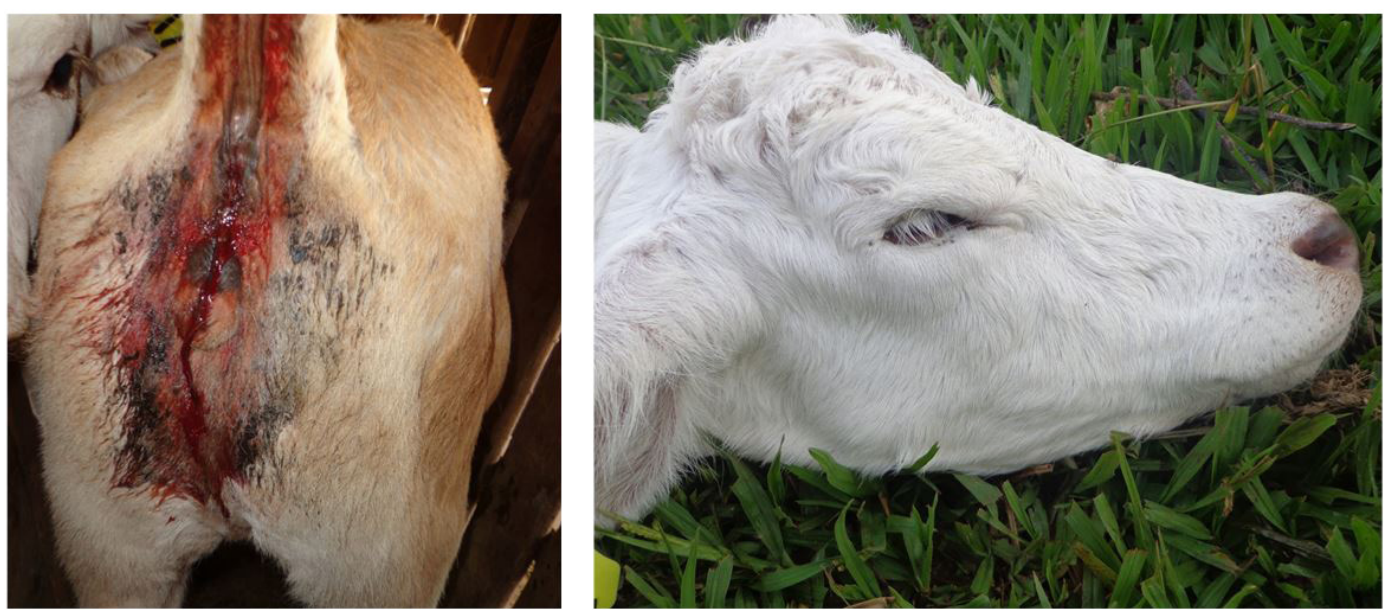

Fig.1. (A) Bezerro apresentando diarreia sanguinolenta desencadeada pelo elevado parasitismo por Eimeria spp. (OoPG 61.550; 99\% de parasitismo por Eimeria zuernii). (B) Bezerro desidratado, diagnosticado por meio da profundidade do globo ocular.
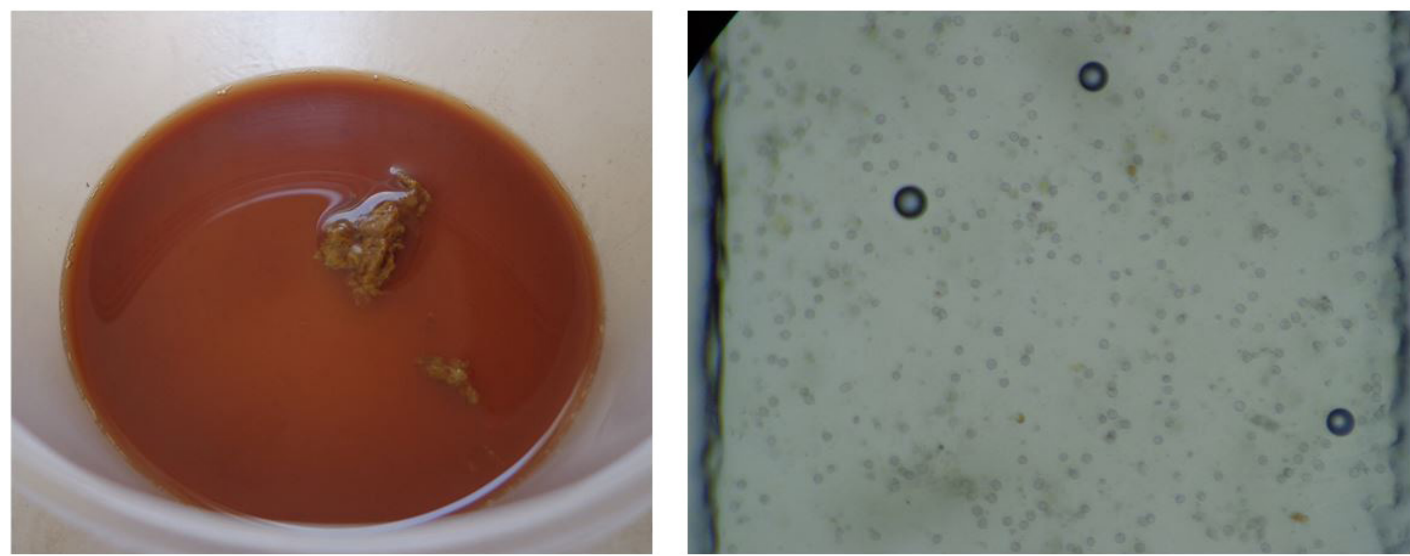

Fig.2. (A) Fragmento de mucosa desprendido juntamente com as fezes de um bezerro com elevado parasitismo por Eimeria. (B) Presença de inúmeros oocistos de Eimeria spp. visualizados na câmera de McMaster durante o exame coproparasitológico. 
primeiro surto foi diagnósticado, uma vez que os animais no $14^{\circ}$ e 28음 DPresentaram contagens médias de OoPG para Eimeria spp. de 14,74 e 123,37, respectivamente (Quadro 2).

Neste primeiro surto, foi possível identificar três espécies de Eimeria spp. parasitando os bezerros. Dentre estas, a mais frequente foi E. zuernii (99,02\%), seguida de E. ellipsoidalis $(0,81 \%)$ e $E$. wyomingensis $(0,17 \%)$. Nas datas subsequentes pós-tratamento dos bezerros com toltrazuril (14 e 28 DPT), houve predomínio de $100 \%$ da espécie E. wyomingensis (Quadro 3).

Nas vacas, a infecção por Eimeria spp. foi quase ausente, uma vez que nos dias zero (primeiro dia da visita no primeiro surto), 14 e 28, as contagens médias de OoPG foram de 1,92 (apenas 2 animais infectados), 1,28 (apenas 3 animais infectados) e 1,92 (apenas 3 animais infectados), respectivamente (Quadro 2). Neste caso, foram identificadas apenas duas espécies, sendo: E. brasiliensis $(66,67 \%)$ e E. canadensis $(33,33 \%)$ no dia zero do estudo (Quadro 3).

Quando analisa-se os bezerros clinicamente durante os 28 dias pós-tratamento, pode-se observar que logo no primeiro dia após a administração do toltrazuril, não foi mais possível visualizar a presença de sangue nas fezes. Por outro lado, todos os animais que apresentavam fezes sanguinolenta, antes do tratamento, continuaram a apresentar fezes diarreicas por até quatro dias após o tratamento (Figura 3AB). Dois bezerros necessitaram receber,trêsvezes,tratamneto suporte (entre o 2o e 4o DPT), de soro com vitamina B12 via endovenosa. Após o 6ํㅡㄹ, não foi possível mais visualizar sinais clínicos sugestivos de eimeriose nos bezerros (Quadro 4).

Quadro 3. Frequência das espécies de Eimeria identificadas nos bezerros e vacas nos primeiro e no segundo surto

\begin{tabular}{|c|c|c|c|c|}
\hline \multirow{3}{*}{ Lote } & \multirow{3}{*}{ Espécie de Eimeria } & \multicolumn{3}{|c|}{ Dia do estudo/Frequência (\%) das espécies de Eimeria identificadas } \\
\hline & & Dia 0 (dia do início do surto) & 14 & 28 \\
\hline & & Contagens médias & Contagens médias & Contagens médias \\
\hline \multicolumn{5}{|c|}{ 1은 Surto diagnosticado em agosto de 2015} \\
\hline \multirow[t]{3}{*}{ Bezerros } & E. zuernii & 99,0 & 0,0 & 0,0 \\
\hline & E. ellipsoidalis & 0,8 & 0,0 & 0,0 \\
\hline & E. wyomingensis & 0,2 & 100,0 & 100,0 \\
\hline \multirow[t]{2}{*}{ Vacas } & E. brasiliensis & 66,7 & 0,0 & 100,0 \\
\hline & E. canadensis & 33,3 & 100,0 & 0,0 \\
\hline \multicolumn{5}{|c|}{$2^{\circ}$ S Surto diagnosticado em agosto de 2015} \\
\hline \multirow[t]{4}{*}{ Bezerros } & E. zuernii & 87,7 & NR & NR \\
\hline & E. subspherica & 7,5 & NR & NR \\
\hline & E. ellipsoidalis & 2,9 & NR & NR \\
\hline & E. wyomingensis & 1,9 & NR & NR \\
\hline \multirow[t]{3}{*}{ Vacas } & E. bovis & 45,7 & NR & NR \\
\hline & E. brasiliensis & 52,8 & NR & NR \\
\hline & E. canadensis & 1,5 & NR & NR \\
\hline
\end{tabular}
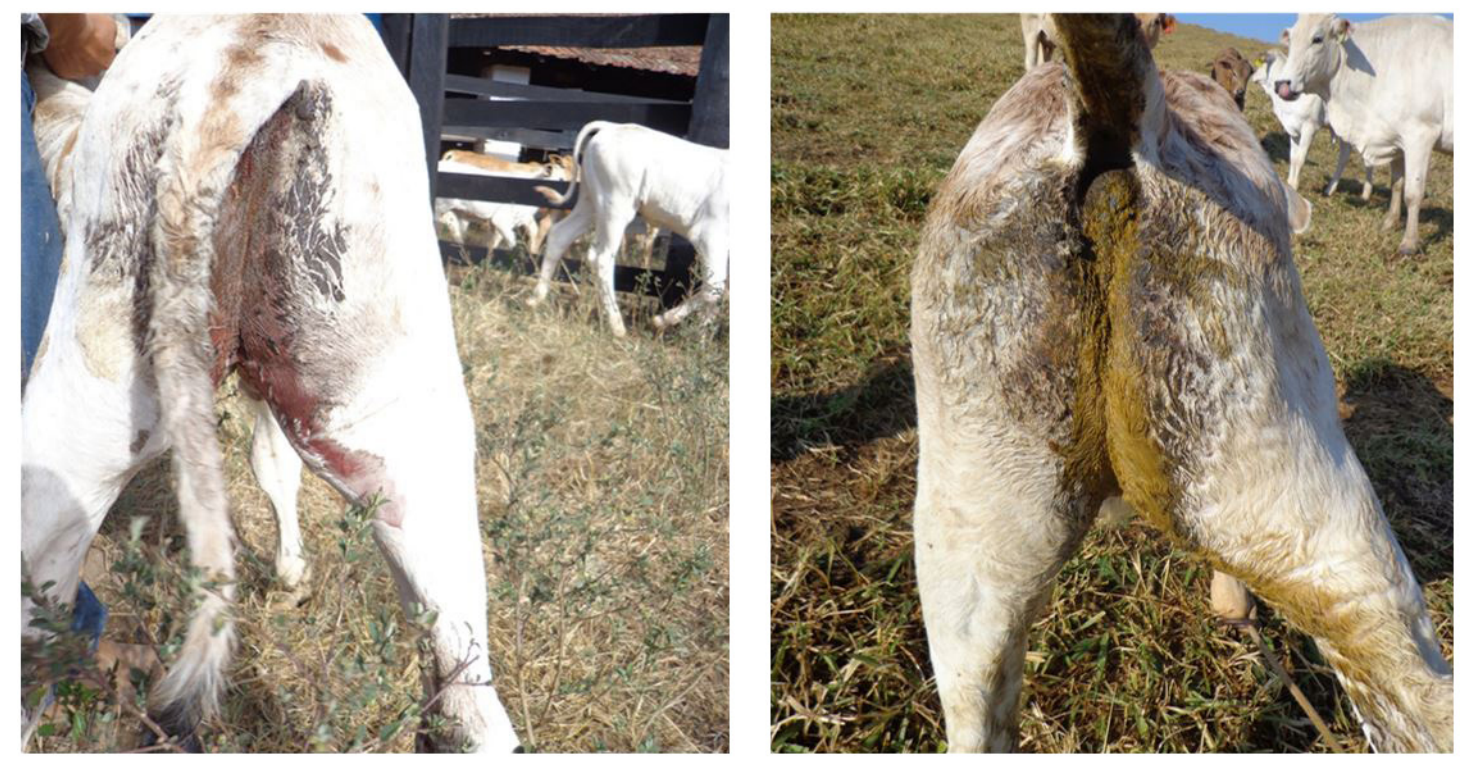

Fig.3. (A) Bezerro com diarreia sanguinolenta antes do tratamento com toltrazuril 15mg/kg. (B) Mesmo bezerro com diarreia (sem a presença de sangue) 3 dias após o tratamento com toltrazuril $15 \mathrm{mg} / \mathrm{kg}$. 
Quadro 4. Histórico relacionado aos surtos diagnosticados em 2015 e 2016

\begin{tabular}{|c|c|c|c|c|}
\hline Número de bezerros no lote & $\begin{array}{l}\text { Número de bezerros } \\
\text { apresentando diarreia } \\
\text { sanguinolenta }\end{array}$ & $\begin{array}{c}\text { Número de bezerros } \\
\text { apresentando diarreia } \\
\text { sanguinolenta e presença de } \\
\text { mucosa intestinal nas fezes }\end{array}$ & $\begin{array}{l}\text { Número de animais } \\
\text { com quadro clínico de } \\
\text { desidratação }\end{array}$ & Mortalidade \\
\hline 78 & $8(10,2 \%)$ & $1(1,3 \%)$ & $2(2,5 \%)$ & $0(0,0 \%)$ \\
\hline \multicolumn{5}{|c|}{ Histórico do surto ocasionado por Eimeria zuernii diagnosticado em 2016} \\
\hline 66 & $4(6,0 \%)$ & $0(0,0 \%)$ & $2(3,0 \%)$ & $2(3,0 \%)$ \\
\hline
\end{tabular}

Segundo surto. Da mesma forma que o ocorrido em agosto de 2015 no segundo surto (agosto de 2016), as contagens médias de OPG encontradas nos bezerros, com aproximadamente 30 dias de idade, foi de 5,30, sendo que apenas três animais demonstraram contagens de OPG entre 50 e 150 (Quadro 1). Neste caso, foram encontrados apenas dois gêneros de helmintos na coprocultura (Haemonchus $76,5 \%$ e Cooperia $23,5 \%$ ). Por outro lado, $39,39 \%$ dos animais (26) encontravam-se infectados por Eimeria spp. Estes mesmos animais apresentaram contagens médias de OoPG de 4.308,93 para o protozoário em questão (Quadro 2). Foram identificadas quatro espécies do referido protozoário, em ordem decrescente: E. zuernii $(87,7 \%)$, E. subspherica $(7,5 \%)$, E. ellipsoidalis $(2,9 \%)$ e E. wyomingensis $(1,9 \%)$ no dia zero (Quadro 3).

Os resultados das contagens médias de OPG $(42,42)$ e OoPG $(6,06)$ encontradas nas vacas neste segundo surto (agosto de 2016), foram semelhantes aos encontrados para esta mesma categoria animal em agosto de 2015 (Quadros 1 e 2). Larvas de Haemonchus (43,7\%), Cooperia (38,9\%), Oesophagostomum $(12,5 \%)$ e Trichostrongylus $(4,9 \%)$ foram encontradas na coprocultura. Como espécies de Eimeria spp., das quatro vacas que apresentaram OoPG entre 50 e 150 , foram identificadas, em ordem decrescente: E. bovis (45,7\%), E. brasiliensis (52,8\%) e E. canadensis (1,5\%) no dia zero (Quadro 3).

A presença de sangue nas fezes cessou um dia após o tratamento dos bezerros com toltrazuril. Por outro lado, fezes amolecidas sem a presença de sangue foi visualizado até o 3 DPT. Tratamento suporte, soro + vitamina B12, foram realizados em dois animais, entretanto, estes dois bezerros vieram à óbito seis dias após eles terem recebido toltrazuril (Quadro 4).

\section{DISCUSSÃO}

Eimeria zuernii e E. bovis são consideradas as espécies mais patogênicas, capazes de desencadearem nos bovinos severa diarréia, com a presença de sangue/fibrina, febre, dor, tenesmo, anemia, desidratação, anorexia e perda de peso (Daugschies \& Najdrowski 2005). Pesquisadores relatam que a forma assintomática é mais comum de ser encontrada em animais adultos, sendo os jovens, de três a seis meses, os mais susceptíveis a formas clínicas da doença (Chibunda et al. 1997, Koutny et al. 2012, Rodrigues 2014). Apesar do exposto acima pelos pesquisadores, é importante ressaltar que no presente trabalho, os surtos ocorreram em bezerros entre 30 a 55 dias de idade e durante a estação seca do ano (agosto). Apesar de que certamente a diarreia nos bezerros tenha ocorrido pelo elevado grau de infecção destes animais por Eimeria zuernii, outras causas, como vírus enteropatogênicos e bactérias, podem ter ocorrido de forma associada.

Outro aspecto que chama atenção é o fato de que, apesar de E. zuernii e E. bovis serem relatadas como as espécies mais prevalentes em bovinos, trabalhos realizados no Brasil, Tanzânia, Turquia, Pakistão, Alemanhã, Irã e Estados Unidos(Rebouças et al. 1994, Chibunda et al. 1997, Cicek et al. 2007, Almeida et al. 2011, Bruhn et al. 2011, Rehman et al. 2011, Bangoura et al. 2012, Bahrami \& Alborzi 2013, Lucas et al. 2014, Rodrigues 2014), reportam que E. bovis foi a espécie mais prevalente nos rebanhos. Tais resultados fizeram com que estes pesquisadores descrevessem $E$. bovis como a mais frequente em bovinos no mundo. Possivelmente o trabalho realizado por Tomczuk et al. (2015) na Polônia, justifique os resultados do presente estudo, em relação a elevada prevalência de E. zuernii diagnosticada. Estes pesquisadores da Polônia reportam que a espécie mais freqüente em bezerros até três ou quatro meses de idade é E. zuernii, enquanto que E. bovis, por sua vez, prevalece em bovinos após esta faixa etária. Nos outros trabalhos realizados nas diversas regiões do mundo, mencionados anteriormente, os animais jovens foram classificados como aqueles desde o nascimento até 12/18 meses de idade, ou, ainda, em outros destes artigos em que a colheita de fezes aconteceu apenas em bovinos com idade superior a $7 / 8$ meses, o que justificaria essa diferença. Em outras palavras, o predomínio de E. zuernii encontrada nos surtos consecutivos reportados neste estudo pode estar relacionado à idade dos animais. De qualquer maneira, futuros estudos relacionados à epidemiologia e/ou de sazonalidade da infecção por espécies de Eimeria spp., em bovinos desde o seu nascimento até oito dez meses de idade, são necessários, a fim de se reforçar ou não os achados do presente estudo e também dos encontrados na Polônia por Tomczuk et al. (2015).

No que diz respeito aos surtos provocados por E. zuernii nos bezerros, é importante destacar que provavelmente as vacas não foram a fonte de infecção para os animais jovens, uma vez que a referida espécie de Eimeria spp., não foi encontrada nas vacas. Além disso, é importante destacar que a frequencia das espécies de Eimeria spp diagnosticadas nas vacas deste estudo, não deve ser levada em consideração no que diz respeito à compararmos ou discutirmos os resultados encontrados com de outras pesquisas, uma vez que tanto o número de vacas infectadas, quanto à carga parasitária pelo coccídio em questão encontrada nestes animais, foi ínfimo.

Lima (2004) relata que apesar de existirem diversos fatores que podem alterar a incidência ou a patogenicidade 

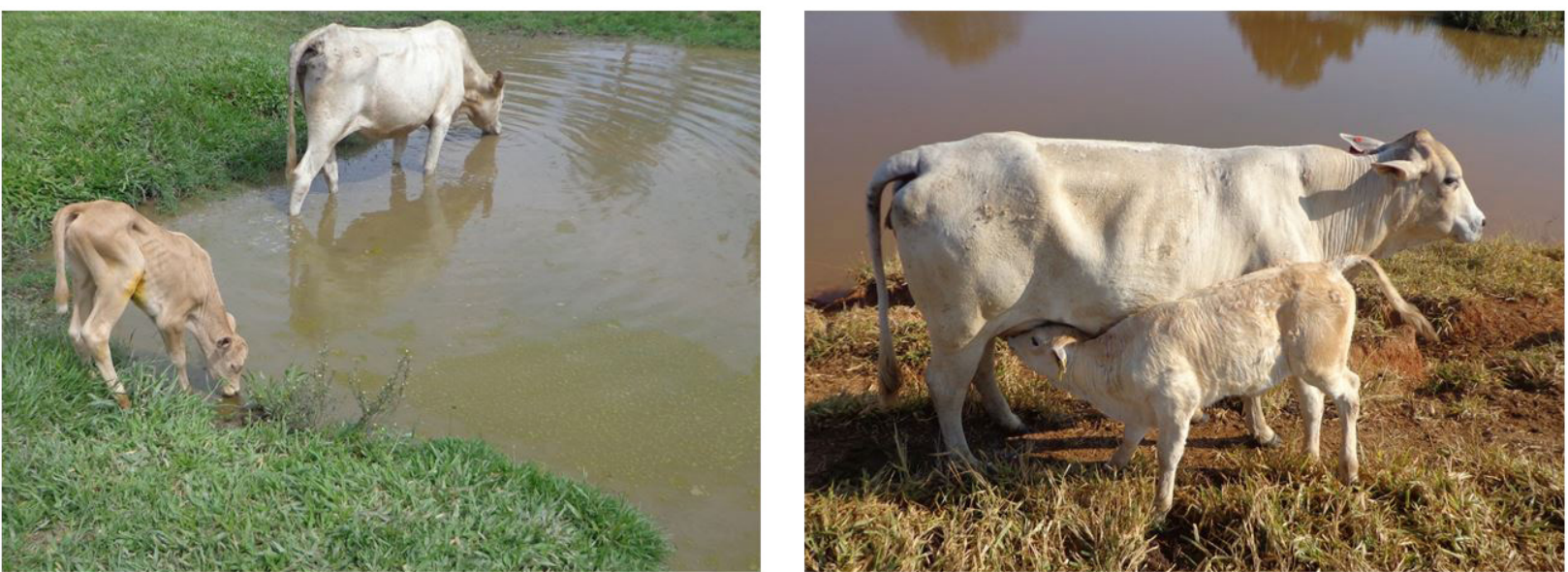

Fig.4. (A) Imagem demonstrando a possível ingestão de oocistos esporulados por meio de água contaminada, no pasto onde os surtos ocorreram. (B) Bezerro amamentando demonstrando possivelmente a ingestão de oocistos esporulados que por ventura se aderiram nas tetas das vacas.

da infecção, em criações extensivas, as áreas onde acumulam a água das chuvas (cacimbas), utilizadas como bebedouros pelos animais, também podem servir como fonte de infecção para oocistos de Eimeria spp. No presente estudo, é importante destacar que a água para os animais beberem era proveniente de nascentes que eram represadas para haver a formação de lagoas no pasto. Em função da idade dos bezerros deste estudo se alimentarem de leite materno (Vaz et al. 2011) e água, muito provavelmente a infecção destes animais por Eimeria spp. aconteceu por meio da ingestão de água diretamente, ou, indiretamente, quando, por ventura, oocistos esporulados se aderiram nas tetas das vacas, quando estas adentraram estas lagoas para beberem água, e quando os bezerros foram amamentar acabaram ingerindo a forma infectante deste protozoário (Fig.4AB).

$O$ pesquisador supracitado enfatiza, ainda, que a adoção de manejo reprodutivo, com o intuito de se aumentar a produtividade, como inseminação artificial em tempo fixo, em uma propriedade, pode vir a aumentar o índice de eimeriose no rebanho, dado que tal método permite concentrar os nascimentos, o que por sua vez acaba aumentando o número de cabeças por área em uma determinada época do ano na propriedade. Por outro lado, é importante frisar que no presente estudo, a taxa de lotação animal adotada pela fazenda para a época do ano (seca) foi relativamente baixa, o que não implicaria no aumento do grau de infecção dos animais pelo coccídio em questão. De qualquer maneira, os bezerros eram provenientes de inseminação artificial, e segundo o proprietário e funcionários da fazenda, o início deste tipo de sinais clínicos começou a aparecer nos bezerros depois de aproximadamente seis anos que esta tecnologia foi adotada na fazenda.

\section{CONCLUSÕES}

Com base nos resultados encontrados neste estudo, é possível afirmar que os surtos consecutivos em bezerros de corte entre 30 e 55 dias de idade, na época seca do ano (agosto), foram desencadeados pelo elevado grau de infecção destes animais por Eimeria zuernii.
Analisando os dois anos em que os surtos ocorreram, a presença de sinais clínicos (diarreia sanguinolenta) e a mortalidade de bezerros desencadeada pela eimeriose/desidratação, aconteceram em 8,3\% 1,4\% do rebanho, respectivamente.

Estes resultados destacam a importância em se realizar pesquisas de campo, com o objetivo de se melhor entender a dinâmica de parasitismo de E. zuernii em bezerros com idade inferior a três meses, em função dos motivos já discutidos neste estudo.

É necessário que se investigue as possíveis fontes de infecção, como a água ingerida pelos animais, para que condutas práticas possam ser adotadas/recomendadas aos proprietários, diminuindo desta maneira os prejuízos ocasionados por surtos de Eimeria spp. em rebanhos bovinos.

\section{REFERÊNCIAS}

Almeida V.A., Magalhães V.C.S., Muniz Neta E.S. \& Munhoz A.D. 2011. Frequency of species of the Genus Eimeria in naturally infected cattle in Southern Bahia, Northeast Brazil. Rev. Bras. Parasitol. Vet. 20(1):78-81. PMid:21439239. http://dx.doi.org/10.1590/S1984-29612011000100017.

Bangoura B., Mundt H.C., Schmäschke R., Westphal B. \& Daugschies A. 2012 Prevalence of Eimeria bovis and Eimeria zuernii in German cattle herds and factors influencing oocyst excretion. Parasitol. Res. 110(2):875-881. PMid:21808979. http://dx.doi.org/10.1007/s00436-011-2569-z.

Bahrami S. \& Alborzi A.R. 2013. Prevalence of subclinical coccidiosis in river buffalo calves of southwest of Iran. Acta Parasitol. 58(4):527-530. PMid:24338314. http://dx.doi.org/10.2478/s11686-013-0167-1.

Bruhn F.R.P., Lopes M.A., Demeu F.A., Perazza C.A., Pedrosa M.F. \& Guimarães A.M. 2011. Frequency of species of Eimeria in females of the holsteinfriesian breed at the post-weaning stage during autumn and winter. Rev. Bras. Parasitol. Vet. 20(4):303-307. PMid:22166384. http://dx.doi. org/10.1590/S1984-29612011000400008.

Chibunda RT., Muhairwa A.P., Kambarage D.M., Mtambo M.M.A., Kusiluka L.J.M. \& Kazwala R.R. 1997. Eimeriosis in dairy cattle farms in Morogoro municipality of Tanzania. Prev. Vet. Med. 31(3-4):191-197. PMid:9234443. http://dx.doi.org/10.1016/S0167-5877(96)01131-2.

Cicek H., Sevimli F., Kozan E., Köse M., Eser M. \& Dogan N. 2007. Prevalence of coccidia in beef cattle in western Turkey. Parasitol. Res. 101(5):12391243. PMid:17653768. http://dx.doi.org/10.1007/s00436-007-0627-3. 
Costa A.J. 1982. Diagnóstico laboratorial em Parasitologia. I. Helmintologia. FCAV-Unesp, Jaboticabal/SP. 89p.

Daugschies A.\& Najdrowski M. 2005. Eimeriosis in cattle: current understanding. J. Vet. Med. B Infect. Dis. Vet. Public Health 52(10):417-427. PMid:16364016. http://dx.doi.org/10.1111/j.1439-0450.2005.00894.x.

Gordon H.Mc.L. \& Whitlock H.V. 1939. A new technique for counting nematode eggs in sheep feces. CSIRO 12:50-52.

Keith R.K. 1953. The differentiation of thei nfective larvae of some common nematode parasites of cattle. Aust. J. Zool. 1(2):223-235. http://dx.doi. org/10.1071/Z09530223.

Koutny H., Joachim A., Tichy A. \& Baumgartner W. 2012. Bovine Eimeria species in Austria. Parasitol. Res. 110(5):1893-1901. PMid:22167365. http://dx.doi.org/10.1007/s00436-011-2715-7.

Lima J.D. 2004. Coccidiose dos ruminantes domésticos. Rev. Bras. Parasitol. Vet. 13:913.

Lopes W.D.Z., Carvalho R.S., Pereira V., Martinez A.C., Cruz B.C., Teixeira W.F., Maciel W.G., Costa A.J., Soares V.E., Borges D.G.L., Rodriguez FS. \& de Almeida Borges F. 2014. Efficacy of sulfadoxine + trimethoprim compared to management measures for the control of Eimeria parasitism in naturally infected and clinically asymptomatic sheep that were maintained in a feedlot. Small Rumin. Res. 116(1):37-43. http://dx.doi.org/10.1016/j. smallrumres.2013.09.010.

Lucas A.S., Swecker Jr W.S., Lindsay D.S., Scaglia G., Neel J.P.S., Elvinger F.C. \& Zajac A.M. 2014. A study of the level and dynamics of Eimeria populations in naturally infected, grazing beef cattle at various stages of production in the Mid-Atlantic USA. Vet. Parasitol. 202(3-4):201-206. PMid:24680603. http://dx.doi.org/10.1016/j.vetpar.2014.02.053.

Matjila P.T. \& Penzhorn B.L. 2002. Occurrence and diversity of bovine coccidian at three localities in South Africa. Vet. Parasitol. 104(2):93-102. PMid:11809329. http://dx.doi.org/10.1016/S0304-4017(01)00605-7.
Rebouças M.M., Grasso M.P.S., Spósito Filha E., Amaral V., Santos S.M. \& Silva D.M. 1994. Prevalência e distribuição de protozoários do gênero Eimeria (Apicomplexa: Eimeriidae) em bovinos nos municípios de Altinópolis, Taquaritinga, São Carlos e Guairá, Estado de São Paulo, Brasil. Rev. Bras. Parasitol. Vet. 3:125-130.

Rehman T.U., Khan M.N., Sajid M.S., Abbas R.Z., Arshad M., Iqbal A. \& Iqbal A. 2011. Epidemiology Eimeria and associated risk factors in cattle of district Toba Tek Singh, Pakistan. Parasitol. Res. 108(5):1171-1177. PMid:21110042. http://dx.doi.org/10.1007/s00436-010-2159-5.

Roberts F.H.S. \& O'Sullivan S.P. 1950. Methods for eggs counts and larva cultures for strongyles infesting the gastrointestinal tract of cattle. Aust. J. Agric. Res. 1(1):99-102. http://dx.doi.org/10.1071/AR9500099.

Rodrigues F.Z. 2014. Eimeria spp. em ruminantes domésticos no Estado de Mato Grosso do Sul. Dissertação de Mestrado, Universidade Federal do Mato Grosso do Sul, Campo Grande, MS.

SAS 2002. SAS ${ }^{\circledR}$ User's Guide: Etatistics. SAS Institute Inc., Cary, NC, USA.

Taubert A., Hermosilla C., Suhwold A. \& Zahner H. 2008. Antigen-induced cytokine production in lymphocytes of Eimeriabovis primary and challenge infected calves. Vet. Immunol. Immunopathol. 126(3-4):309320. PMid:18947883. http://dx.doi.org/10.1016/j.vetimm.2008.09.003.

Tomczuk K., Grzybek M., Szczepaniak K., Studzinska M., Demkowska-Kutrzepa M., Roczen-Karczmarz M. \& Klockiewicz M. 2015. Analysis of intrinsic and extrinsic factors influencing the dynamics of bovine Eimeria spp. From central-eastern Poland. Vet. Parasitol. 214(1-2):22-28. PMid:26455571. http://dx.doi.org/10.1016/j.vetpar.2015.09.027.

Ueno H. \& Gonçalves P.C. 1998. Manual para diagnóstico das helmintoses de ruminantes. JICA Newsl. 10:25-28.

Vaz R.Z., Lobato J.P. \& Pascoal L.L. 2011. Desenvolvimento de bezerros de corte desmamados aos 80 ou 152 dias até os 15-16 meses de idade. Rev. Bras. Zootec. 40(1):221-229. http://dx.doi.org/10.1590/S151635982011000100030 . 